Original Paper http://ajol.info/index.php/ijbcs http://indexmedicus.afro.who.int

\title{
Structure et diversité taxonomique des peuplements de macroinvertébrés benthiques du réseau hydrographique du bassin du Sebou (Maroc)
}

\author{
Fatima ABBOU* et Abdelilah FAHDE
}

\author{
Laboratoire d'Ecologie Aquatique et d'Environnement, Département de Biologie, \\ Université Hassan II, Faculté des Sciences Ä̈n Chock Km 8 Route d'El Jadida B.P 5366 Maârif Casablanca \\ 20100 Maroc. \\ *Auteur correspondant ; E-mail: abboufatima@yahoo.fr; Tél : +212641659136.
}

\section{RESUME}

Oued Sebou est le plus important fleuve se jetant dans l'Atlantique. Son bassin connaît une activité industrielle très développée, ainsi qu'une importante activité domestique et agricole ; d'où la nécessité d'évaluer la qualité de ses eaux. L'intensification de ces activités génère une pollution importante entrainant la dégradation globale de l'habitat ainsi que la raréfaction et la mortalité de la biocénose. Des études faunistiques et écologiques (structure des communautés d'invertébrés benthiques, répartition spatiale et abondance relative) ont été effectuées pour comprendre le fonctionnement du bassin et pour évaluer son état de santé écologique. Les macroinvertébrés de ce cours d'eau ont été étudiés à partir des prélèvements réalisés entre mars 2011 et octobre 2011. La faune recensée dans ce travail se compose de 9990 individus correspondant à 52 familles et à 80 taxons appartenant à 3 groupes faunistiques principales (Annélides, Mollusques, arthropodes). L'effectif du peuplement benthique a montré que les Ephéméroptères, les Diptères, les Crustacés et les Oligochètes sont dominants. Les Trichoptères, les Gastéropodes, les Coléoptères, les Hétéroptères, les Odonates et les Achètes ne constituent qu'une faible fraction de la faune récoltée. Les taxons Baetis rhodani, Chironomus $s p$, Gammarus sp, Tubifex tubifex sont les plus abondants. L'analyse de la structure biotypologique, de l'Oued Sebou montre que les stations à proximité des agglomérations se caractérisent par une diminution voire une disparition des taxons polluo-sensibles tels que les Plécoptères et les Éphéméroptères, et une prolifération des taxons polluo-tolérants comme les Chironomidae et les Oligochètes.

(C) 2017 International Formulae Group. All rights reserved.

Mots clés : Sebou, macroinvertébrés, biotypologie, polluo-sensible, polluo-tolérant.

\section{Structure and taxonomic diversity of benthic macrofauna of the hydrographic network of the Sebou River (Morocco)}

\section{ABSTRACT}

Sebou is the most important river flowing into the Atlantic. Its basin knows important industrial, domestic and agricultural activities; hence the need to assess the quality of its waters. The intensification of 
these activities generates important pollution causing global habitat degradation and rarefaction and mortality of the biocenosis. Faunistic and ecological studies (benthic macroinvertebrates communities, spatial distribution, community structure and relative abundance) have been done to understand the functioning of the river basin and to evaluate its ecological status. Macroinvertebrates of the Sebou River were studied from samples collected from March 2011 to November 2011. The fauna recorded in this work is composed of 9990 individuals corresponding to 52 families and 80 taxa belonging to three faunistic groups (annelids, molluscs, arthropods). The number of benthic population showed that Ephemeroptera, Diptera, Crustaceans and Oligochaeta, are dominant. Trichoptera, Gastropods, Coleoptera, Heteroptera, Odonata and the Hirudinea are only a small fraction of the total fauna. Taxa Baetis rhodani, Chironomus sp, Gammarus sp, Tubifex tubifex are numerically more inventoried. Analysis of the biotypology structure of the Sebou River shows that more we advance downstream, more we are seeing a decrease or disappearance of taxa polluo-sensitive such as Plecoptera and Ephemeroptera. The opposite, settled for polluo-tolerant taxa such as Chironomidae and Oligochaeta.

(C) 2017 International Formulae Group. All rights reserved.

Keywords: Sebou, macroinertebrates, biotypology, polluo-sensitive, polluo-tolerant.

\section{INTRODUCTION}

L'évaluation de la qualité des eaux et le suivi des impacts des activités anthropiques sur les cours d'eau font généralement appel à la mesure de paramètres physico-chimiques et écotoxicologiques de l'eau et du sédiment. Cette approche a montré leur utilité mais aussi a ses limites (Beyene et al., 2009). Les méthodes biologiques, comme les indices basés sur la macrofaune invertébrée, constitue selon Foto Menbohan (2013) la seule voie valable pour l'appréciation générale de la qualité des systèmes et donc des effets réels de la pollution, la notion d'intégrité ou santé des écosystèmes nécessite de prendre en compte simultanément les paramètres chimiques, physiques et biologiques (Colas et al., 2014).

Les études faunistiques et écologiques revêtent d'une importance primordiale dans la compréhension du fonctionnement et de la gestion des systèmes naturels d'une part, et d'autre part, dans l'évaluation de l'état de santé écologique des hydrosystèmes (Kaboré et al., 2016). Les macroinvertébrés benthiques sont considérés comme de très bons indicateurs biologiques En effet, ils sont relativement sédentaires, abondants et relativement faciles à collecter. Leurs communautés sont capables de présenter un gradient caractéristique de réponses selon l'intensité et la nature du stress. De plus, leur durée de vie est suffisamment longue et elles sont taxonomiquement très hétérogènes et diversifiées. En outre, leur sensibilité est variable et différenciée face aux différents types de polluants (Moisan et al., 2008) et leurs réactions sont généralement rapides pour fournir un enregistrement intégré de la qualité environnementale. Enfin, les macroinvertébrés dans leur ensemble sont ubiquistes dans les réseaux hydrographiques. D'où la nécessité d'étudier les macroinvertébrés benthiques afin de les utiliser comme outil d'évaluation de la qualité des eaux. L'objectif de ce travail était donc d'étudier la diversité taxonomique et la structure de la macrofaune benthique du réseau hydrographique du bassin du Sebou afin d'évaluer la qualité écologique de ses eaux.

\section{MATERIEL ET METHODES \\ Zone d'étude}

L'Oued Sebou, ayant une longueur totale de $614 \mathrm{~km}$ depuis sa source, est situé au Nord-Ouest du Maroc, entre 4-7 degré Ouest 
et 33-35 degré Nord. Le bassin de Sebou forme une cuvette entre le Rif au Nord, le moyen Atlas et la méseta au Sud, le couloir Fès-Taza à l'Est et l'océan Atlantique à l'Ouest. Le climat régnant sur l'ensemble de l'Oued Sebou est de type méditerranéen à influence océanique et devient continental vers l'intérieur. Les précipitations annuelles moyennes sur l'ensemble du bassin du Sebou, sont de $600 \mathrm{~mm}$ environ.

L'étude de la macrofaune des cours d'eau du bassin du Sebou ne pouvait être entreprise sur l'ensemble du réseau hydrographique du fleuve. Il était donc nécessaire d'avoir un nombre de stations de prélèvement suffisamment élevé. Plusieurs compagnes de terrain ont été réalisées entre mars 2011 et octobre 2011. Pour réaliser et mener à bien cette étude, vingt-six stations ont été choisies en tenant compte d'un certain nombre de critères : diversité lithologique du bassin, situation des points de prélèvement par rapport aux rejets polluants, proximité des agglomérations rurales, facilités d'accès et de prélèvement. Ces stations sont réparties, de l'amont vers l'aval, selon un gradient croissant de pollution : 4 stations (S1 à S4) au niveau du Haut Sebou situées à proximité des sources dans le Moyen Atlas, 12 stations (S5 à S16) au niveau du Moyen Sebou à proximité des sources de pollution et 10 stations (S17 à S26) au niveau du Bas Sebou éloignées des sources de pollution.

La Figure 1 et le Tableau 1 montrent les vingt-six stations représentatives de l'ensemble des biotopes échantillonnés.

\section{Echantillonnage et analyse}

A chaque station d'une longueur égale à environ dix fois la largeur du cours d'eau, le nombre de prélèvements effectués est de douze prélèvements. Ces prélèvements ont été réalisés à différents endroits présentant des couples "substrat-vitesse de courant" différents. Chaque prélèvement est considéré comme représentatif de la faune benthique sur une surface de $1 / 20 \mathrm{~m}^{2}$.

$\mathrm{Au}$ sein d'une station, les principaux types de faciès ont été prospectés: Les secteurs lotiques ont été échantillonnés à l'aide d'un filet de type Surber, équipé d'un filet d'ouverture de maille de $500 \mu \mathrm{m}$ et pourvu d'une base de surface de $1 / 20 \mathrm{~m}^{2}$. Les secteurs lentiques ont été prospectés à l'aide d'un filet haveneau.

Dans une station, les prélèvements ont été fixés sur le terrain par addition d'une solution de formol à $10 \%$ (Foto Menbohan et al., 2011). Avant de fixer les échantillons, un pré-tri a permis d'enlever les éléments minéraux (cailloux, graviers et sables) car ceux-ci sont susceptibles d'endommager la faune lors du transport (Karrouch, 2010; Haouchine, 2011). Le tri et la détermination des invertébrés ont été effectués en laboratoire au moyen d'une loupe binoculaire (grossissement maximal de 40). Tous les individus ont été comptés. Le traitement des échantillons a nécessité de nombreux mois de travail (7 au total). L'identification des spécimens a été effectuée au niveau taxonomique le plus précis possible (genre ou espèce). La détermination des spécimens récoltés est réalisée en faisant appel à des ouvrages, collections et clés de détermination des macroinvertébrés (Poisson, 1957; Clergue et al., 1991; Dommanget, 1994; Tachet et al., 2010). 


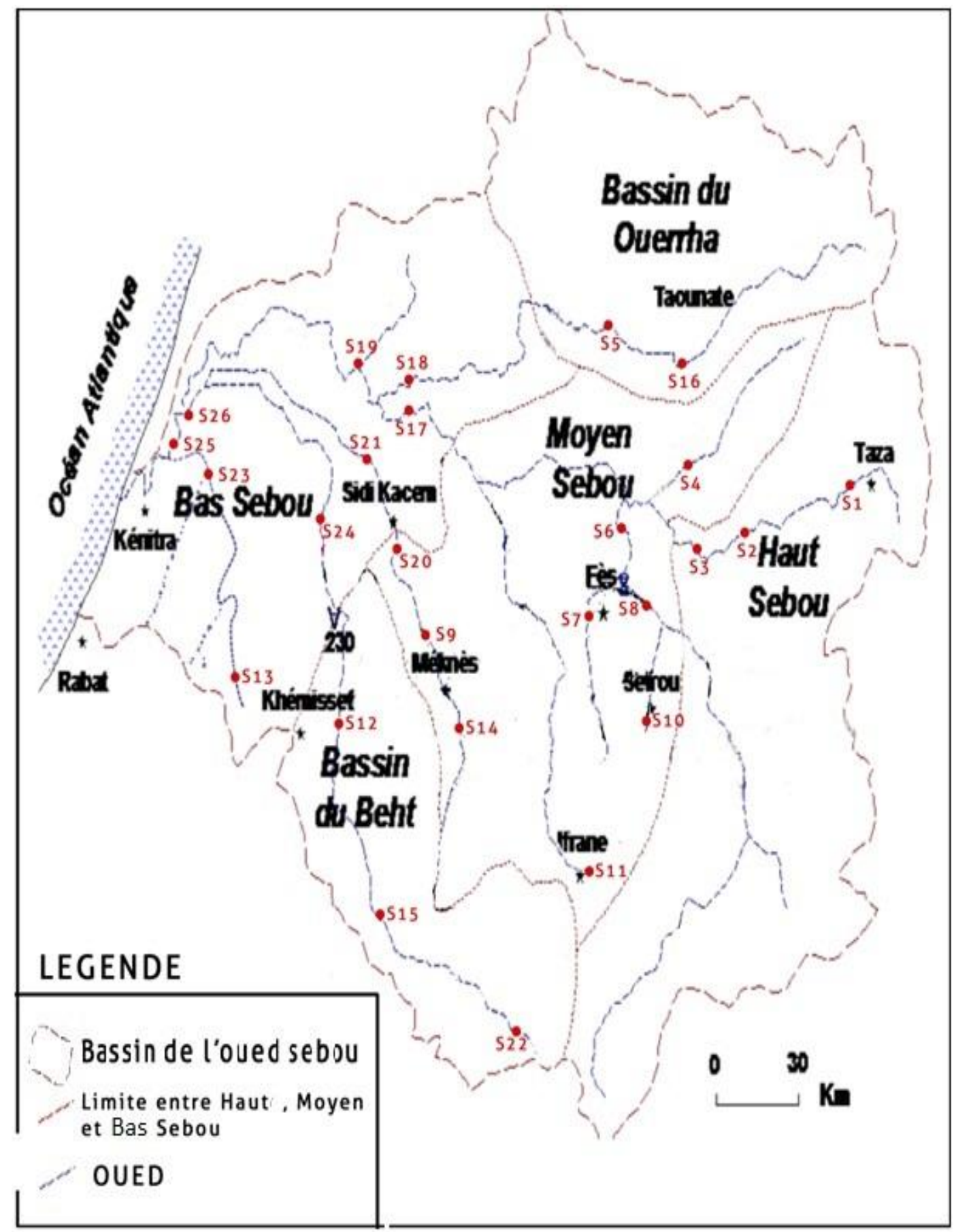

Figure 1 : Carte de localisation des stations de prélèvement. 
Tableau 1 : Localisation, coordonnées et caractéristiques des stations d'étude.

\begin{tabular}{|c|c|c|c|c|c|c|}
\hline Stations & Code & Province & Commune & $\begin{array}{c}\text { Coordonnées } \\
\text { lambert }\end{array}$ & Profondeur (m) & Débit (1/s) \\
\hline Puits RP1 & $\mathrm{S} 1$ & Taza & $\begin{array}{c}\text { Bab } \\
\text { Marzouka }\end{array}$ & $\begin{array}{c}\mathrm{X}: 623,25 \\
\mathrm{Y}: 404,600\end{array}$ & 0,35 & Moyen \\
\hline Bab Marzouka & $\mathrm{S} 2$ & Taza & $\begin{array}{c}\text { Bab } \\
\text { Marzouka }\end{array}$ & $\begin{array}{c}X: 615,85 \\
Y: 400,850\end{array}$ & 10,35 & Moyen \\
\hline Station El Kouchet & $\mathrm{S} 3$ & Taza & $\begin{array}{c}\text { Bab } \\
\text { Marzouka }\end{array}$ & $\begin{array}{c}X: 583,75 \\
Y: 394,650\end{array}$ & 0,15 & Moyen \\
\hline PT RS 302 (Tissa) & S4 & Taounate & Tissa & $\begin{array}{l}X: 558,250 \\
Y: 405,950\end{array}$ & - & - \\
\hline Rhafsai & S5 & Taounate & Tissa & $\begin{array}{c}\mathrm{X}: 542,84 \\
\mathrm{Y}: 445,940 \\
\end{array}$ & - & - \\
\hline Pont Portugais & S6 & $\begin{array}{c}\text { Fès } \\
\text { Médina }\end{array}$ & Fès Médina & $\begin{array}{c}X: 544,05 \\
Y: 385,825\end{array}$ & - & - \\
\hline Aval rejet de Fès & S7 & $\begin{array}{c}\text { Fès } \\
\text { Médina }\end{array}$ & Fès Médina & $\begin{array}{c}\mathrm{X}: 543,38 \\
\mathrm{Y}: 386,540\end{array}$ & 0,2 & Moyen \\
\hline Dar El Arsa & S8 & Fès & Loudayene & $\begin{array}{l}X: 543,300 \\
Y: 399,700\end{array}$ & 0,25 & Moyen \\
\hline Pont Kroumam & S9 & Meknès & $\begin{array}{c}\text { Oued } \\
\text { Romane }\end{array}$ & $\begin{array}{l}X: 466,900 \\
Y: 410,800\end{array}$ & 0,25 & Moyen \\
\hline Aval Sefrou & $\mathrm{S} 10$ & Taza & $\begin{array}{c}\text { Bab } \\
\text { Marzouka }\end{array}$ & $\begin{array}{c}X: 554,27 \\
Y: 365,850\end{array}$ & - & - \\
\hline Sidi Mokhfi & S11 & Ifrane & $\begin{array}{l}\text { Sidi El } \\
\text { Mokhfi }\end{array}$ & $\begin{array}{l}X: 507,600 \\
Y: 311,800\end{array}$ & 0,7 & Fort \\
\hline $\begin{array}{l}\text { Aval rejet } \\
\text { Khemisset }\end{array}$ & $\mathrm{S} 12$ & $\begin{array}{c}\text { Khemisse } \\
\mathrm{t}\end{array}$ & Tifelt & $\begin{array}{c}\mathrm{X}: 450,30 \\
\mathrm{Y}: 369,150 \\
\end{array}$ & - & - \\
\hline Aval rejet Tifelt & $\mathrm{S} 13$ & $\begin{array}{c}\text { Khemisse } \\
\mathrm{t}\end{array}$ & Tifelt & $\begin{array}{c}\mathrm{X}: 450,30 \\
\mathrm{Y}: 369,150 \\
\end{array}$ & - & - \\
\hline $\begin{array}{l}\text { Aval rejet de } \\
\text { Meknès }\end{array}$ & S14 & $\begin{array}{c}\text { Khemisse } \\
\mathrm{t}\end{array}$ & Tifelt & $\begin{array}{c}X: 450,30 \\
Y: 369,150\end{array}$ & 30 & Moyen \\
\hline Ouljet Soltane & $\mathrm{S} 15$ & $\begin{array}{c}\text { Khemisse } \\
\mathrm{t}\end{array}$ & Tifelt & $\begin{array}{c}X: 450,30 \\
Y: 369,150 \\
\end{array}$ & - & - \\
\hline Ain Aicha & S16 & $\begin{array}{c}\text { Khemisse } \\
\mathrm{t}\end{array}$ & Tifelt & $\begin{array}{c}\mathrm{X}: 564,70 \\
Y: 428,800\end{array}$ & 0,2 & Moyen \\
\hline Pont Khenichet & S17 & $\begin{array}{c}\text { Sidi } \\
\text { Kacem }\end{array}$ & Khenichet & $\begin{array}{c}\mathrm{X}: 449,35 \\
Y: 515,180\end{array}$ & - & Impotant \\
\hline Had kourt & S18 & $\begin{array}{c}\text { Sidi } \\
\text { Kacem }\end{array}$ & Had Kourt & $\begin{array}{c}\mathrm{X}: 470,35 \\
\mathrm{Y}: 439,900\end{array}$ & - & - \\
\hline $\begin{array}{l}\text { Aval Confluence } \\
\text { Sebou-Rdat }\end{array}$ & S19 & $\begin{array}{c}\text { Sidi } \\
\text { Kacem }\end{array}$ & Sidi Fath & $\begin{array}{c}X: 459,50 \\
Y: 432,100\end{array}$ & Profond & Impotant \\
\hline Azib Soltane & $\mathrm{S} 20$ & $\begin{array}{c}\text { Sidi } \\
\text { Kacem }\end{array}$ & Selfate & $\begin{array}{c}X: 492,00 \\
Y: 413,900\end{array}$ & - & - \\
\hline $\begin{array}{l}\text { RP26 (Kariat Ba } \\
\text { Med) }\end{array}$ & $\mathrm{S} 21$ & $\begin{array}{c}\text { Sidi } \\
\text { Kacem }\end{array}$ & Selfate & $\begin{array}{c}\mathrm{X}: 523,25 \\
\mathrm{Y}: 412,150\end{array}$ & 0,2 & Moyen \\
\hline Souk El Had & $\mathrm{S} 22$ & $\begin{array}{c}\text { Sidi } \\
\text { Kacem }\end{array}$ & Chebanate & $\begin{array}{l}X: 466,900 \\
Y: 410,800\end{array}$ & 0,4 & Moyen \\
\hline
\end{tabular}




\begin{tabular}{lcccccc}
\hline Pont Dar Bel Amri & S23 & Kénitra & $\begin{array}{c}\text { Dar Bel } \\
\text { Amri }\end{array}$ & $\begin{array}{c}\text { X: 447,72 } \\
\text { Y: 398,970 }\end{array}$ & - & - \\
\hline Aval Sidi Slimane & S24 & Kénitra & Ksiba & $\begin{array}{c}\text { X: 449,35 } \\
\text { Y: 515,180 }\end{array}$ & 0,35 & $\begin{array}{c}\text { Faible à } \\
\text { moyen }\end{array}$ \\
\hline Barrage de Garde & S25 & Kénitra & $\begin{array}{c}\text { Ben } \\
\text { Mansour }\end{array}$ & $\begin{array}{c}\text { X: } 407,00 \\
\text { Y: 432,500 }\end{array}$ & - & - \\
\hline $\begin{array}{l}\text { Aval Confluence } \\
\text { Sebou-Beht }\end{array}$ & S26 & Kénitra & Morgana & $\begin{array}{c}\text { X: 404,70 } \\
\text { Y: 423,600 }\end{array}$ & - & - \\
\hline
\end{tabular}

\section{RESULTATS} $\begin{aligned} & \text { Abondance quantitative des groupes } \\ & \text { faunistiques }\end{aligned}$
a

Au total, 9990 individus appartenant à 52 familles et 80 taxons correspondant à 3 groupes faunistiques (Annélides, Mollusques, Arthropodes) ont été inventoriés.

L'abondance de la faune varie considérablement d'une station à une autre, allant de 1 à 1287 individus (Figure 2). Ces variations spatiales pourraient être attribuées aux diverses influences que subit les milieux et aussi à la nature des différents habitats.

L'analyse de l'ensemble du peuplement récolté durant la période d'étude, montre que les insectes sont numériquement les plus inventoriés et représentent le pourcentage le plus élevé $(72,71 \%)$ suivi par les Crustacés $(12,93 \%)$, les Annélides $(8,66 \%)$ et les Mollusques (5,15\%) (Figure 2).

\section{Composition faunistique des peuplements benthiques}

L'inventaire faunistique établi

(Tableau 2) regroupe la répartition des peuplements dans les différentes stations d'étude. Les peuplements benthiques du réseau hydrographique du bassin du Sebou sont caractérisés par une faible diversité spécifique du peuplement benthique, en particulier une faible diversité intragénérique; en effet la plupart des taxons sont monospécifiques.

$\begin{gathered}\text { Evolution spatiale } \\ \text { taxonomique totale }\end{gathered}$
Les effectifs totaux des taxons
inventoriés durant la période d'étude sont variables d'une station à l'autre (Figure 3). En effet, le peuplement des stations (S7, S8, S12 et S13) est le moins diversifié, ne dépassant pas cinq taxons par station. La réduction de la richesse taxonomique s'observe nettement en aval des zones les plus urbanisées et industrialisées soumises aux rejets domestiques et industrielles.

Dans les stations étudiées, les taxons dominants sont les Diptères (essentiellement les Chironomidae) et les Annélides (essentiellement les Oligochètes). Concernant les autres stations, elles montrent globalement une variété taxonomique plus importante (environ 15 taxons par station). La station la plus diversifiée est la station S6 (Pont Portugais) avec un effectif de 26 taxons (Figure 3).

De façon générale, on constate, au niveau des stations étudiées, une dominance des taxons polluo-résistants, principalement les Diptères et les Annélides (Figure 2).

\section{Analyse qualitative et quantitative de la faune benthique \\ Les Achètes}

D'après les résultats obtenus, ces Annélides sont représentés par 3 familles (Glossiphoniidae, Hirudidae et Erpobdellidae) réparties en 8 taxons. Dans ce groupe d'organismes, les Glossiphoniidae sont dominants avec 29 individus (soit 55,77\% des Achètes), ils sont représentés par 2 genres (Glossiphonia et Batrocobdella). Les Erpobdellidae $(32,69 \%$ des Achètes) sont représentés par 3 genres: Erpobdella, Helobdella et Haementeria. Quant aux Hirudidae, ils sont faiblement représentés (6 
individus soit $11,54 \%$ des achètes) (Figure $4 \mathrm{~A})$. La répartition spatiale des Achètes (Figure 4B) montre que ces invertébrés se développent favorablement dans les stations situées à proximité des agglomérations ( $\mathrm{S} 6$, S9, S10 et S11). Cependant, on observe une quasi-absence voire même une absence de ces organismes dans les autres stations. En effet, leur pourcentage ne dépasse pas $0,3 \%$ du total du benthos.

\section{Les Oligochètes}

Ces annélides sont représentés par 3 familles (Haplotaxidae, Lumbricidae et Tubificidae) correspondant à 4 taxons. Ces macroinvertébrés sont dominés par les Tubificidae avec 802 individus de Tubifex tubifex (soit $95,7 \%$ des oligochètes). Les Lumbricidae occupent le deuxième rang avec 24 individus de Eiseniella tetraedra $(2,86 \%$ des oligochètes). Quant aux Haplotaxidae, ils ont une très faible importance numérique (Figure 5A). Concernant la répartition spatiale de ces organismes (Figure 5B), les stations (S7 et S14) respectivement en aval des villes de Fès et de Meknès renferment abondamment d'Oligochètes constitués essentiellement des Tubificidae $(99 \%$ du benthos au niveau de S7 et $20 \%$ au niveau de S14). Par contre, on note une diminution importante du nombre d'individus au niveau des autres stations.

\section{Les Gastéropodes}

Les Gastéropodes sont représentés par 7 familles correspondant à 9 taxons : Physidae, Planorbidae, Viviparidae, Hydrobiidae, Bithyniidae, Amnicolidae et Melanopsidae. Les Physidae sont dominants et comptent 429 individus $(80 \%$ des mollusques) et représentés essentiellement par Physa acuta. Les autres familles ont une très faible importance numérique (Figure 6A). La répartition spatiale des Gastéropodes (Figure 6B) montre qu'ils constituent l'un des groupes d'invertébrés benthiques les plus importants. Leur prolifération semble plus importante surtout dans les stations (S6, S18 et S23) et l'espèce Physa acuta est la plus dominante et occupe toutes les eaux des stations étudiées.

\section{Les Crustacés}

Dans les stations prospectées, 1250 individus (soit $12,51 \% \mathrm{~d}$ la faune totale récoltée) appartenant à 2 familles et 2 genres ont été récoltés: Gammaridae (Gammarus $s p$.) et Atyidae (Atyaephyra sp.). Les Gammaridae sont dominants et comptent plus de 1025 individus (soit $82 \%$ des crustacés) et représentés par un seul genre Gammarus (Figure 7A). La famille Atyidae est représentée par l'espèce Atyaephyra desmarestii et compte 225 individus (soit $18 \%$ ). La répartition spatiale des crustacés (Figure 7B) révèle la présence de ce groupe zoologique dans la majorité des stations du bassin du Sebou. Il est représenté essentiellement par le genre Gammarus, avec un pic maximal en S9 avec une abondance relative égale à 88,36\% (soit 1025 individus).

\section{Les Plécoptères}

Les Plécoptères n'ont pas été récoltés dans les stations prospectées.

\section{Les Trichoptères}

La faune récoltée des Trichoptères comptent 556 individus. Quatre familles ont été récoltées: Hydropsychidae (511 individus), Hydroptilidae (3 individus), Philopotamidae (17 individus) et Polycentropodidae (25 individus). Les individus récoltés se répartissent en 5 genres. Seule la famille des Hydroptilidae est représenté par deux espèces: Hydropsyche maroccana et Hydroptila sp. Les autres familles sont monospécifiques: Orthotrichia $s p$., Ecnomus sp., Chiamarra marginata et Neureclipsis bimaculata. On constate ainsi que la famille la plus abondante est celle des Hydropsychidae $(91,74 \%$ des Trichoptères) (Figure 8A) et qui est représentée par l'espèce la plus commune des cours d'eau marocains : Hydropsyche maroccana (Vernaeux et al, 1976). La distribution spatiale des Trichoptères (Figure 8B) se traduit par une abondance de Hydropsyche maroccana avec un pic maximal en S3, et des abondances relativement importante au niveau des stations S9 et S11. 


\section{Les Hétéroptères}

Ces insectes sont représentés seulement par 82 individus. Ce groupe d'invertébrés appartient à 3 familles correspondant à 3 espèces: Corixidae (Micronecta scholtzi), Notonectidae (Notonecta maculata) et Gerridae (Gerris argentatus). La famille des Corixidae est la mieux représentée parmi les Hétéroptères (78 individus soit $95,12 \%$ de ce groupe faunistique). Cependant, les autres familles sont faiblement représentées: Notonectidae $(2,43 \%)$ et Gerridae $(2,43 \%)$ (Figure $9 \mathrm{~A})$. La répartition spatiale des Hétéroptères (Figure 9B) montre un pic maximal en S6 (100 individus), alors que le nombre des individus récoltés dans les autres stations est très faible. Quant au nombre de genres inventoriés, il est compris entre 1 et 2 genres.

\section{Les Coléoptères}

Les Coléoptères constituent un groupe plus au moins diversifié par rapport aux autres groupes. Au total, le nombre de genres inventoriés est de l'ordre de 13. Les deux genres les plus représentés sont Gyrinus $s p$. (261 individus soit 65,08\%) et Laccophilus sp. (75 individus soit 18,7\%) (Figure 10A). Pourtant, malgré la diversité taxonomique de ce groupe zoologique, les abondances restent dans l'ensemble faibles. La distribution spatiale des Coléoptères (Figure 10B) montre globalement la présence de ces invertébrés dans toutes les stations avec un effectif important au niveau des stations (S21, S15) et un effectif faible au niveau des autres stations. Concernant la richesse taxonomique des stations en Coléoptères, on note un pic maximal au niveau de S15. Ceci pourrait être dû au fait que les biotopes de cette station sont hétérogènes, ce qui favorise la prolifération de ce groupe d'invertébrés. En revanche, la variété taxonomique varie entre 1 et 4 dans les autres stations caractérisées par la présence de ce groupe.

\section{Les Ephéméroptères}

Ces insectes constituent le groupe faunistique le plus abondant. Ils comptent 3536 individus, soit $35,39 \%$ de la faune totale récolté dans le bassin du Sebou. Les Ephéméroptères constituent un groupe plus au moins diversifié. En effet, les individus récoltés sont répartis en 11 genres appartenant à 5 familles (Figure 11A). Sur le plan de la diversité taxonomique, La famille Baetidae, avec 5 espèces recensées (Baetis rhodani, Baetis fuscatus, Acentrella sinaica, Centroptilum sp. et Procloeon sp.), est la plus diversifiée parmi les Ephéméroptères récoltés. Elle est égalament la plus abondante, elle représente $76,86 \%$ de ce peuplement, soit 2341 individus. Les Caenidae (représentés par une seule espèce) et les Heptageniidae (représentés par 4 espèces) occupent le deuxième et le troisième rang sur le plan d'abondance numérique. Ces deux familles constituent respectivement $15,41 \%$ et $7,35 \%$. Les autres familles, représentées uniquement par une seule espèce, sont faiblement représentées: Leptophlebiidae $(0,25 \%)$ et Oligoneuriidae $(0,11 \%)$. La répartition spatiale des Ephéméroptères (Figure 11B) montre que ce groupe d'insectes occupent presque la totalité des milieux étudiés. Les abondances maximales des Ephéméroptères ont été observées en S11 (663 individus soit $48,99 \%$ de la faune totale récoltée dans cette station), S14 (625 individus soit $69,91 \%$ ) et S17 (575 individus soit 79,09\%). Ces maxima d'abondance coïncident avec la prolifération de la population de Baetis rhodani (espèce méditerranéenne) et Caenis luctuosa (espèce de la zone paléarctique occidentale). Aussi, la richesse taxonomique dans ces stations est importante ( 7 à 8 espèces). En revanche, on note une diminution de l'abondance de ce groupe zoologique au niveau des autres stations.

\section{Les Diptères}

Les Diptères constituent le groupe taxonomique le plus diversifié du réseau hydrographique du bassin du Sebou. En effet, dans les échantillons récoltés, ces insectes comptent 2316 individus appartenant à 14 familles dont l'abondance diffère d'une famille à une autre (Figure 12A). Les familles les plus importantes sont celles des 
Chironomidae et des Simuliidae. La Famille des Chironomidae est représentée par 10 taxons regroupés en 2 sous-familles: les Chironominae et les Orthocladiinae. Quant aux Simuliidae, ils sont représentés par 2 espèces: Whilhelmia pseudoquinum et Metacnephia blanci. La répartition spatiale des Diptères (Figure 12B) montre que ces invertébrés sont omniprésents dans toutes les stations des cours d'eau étudiés mais avec des proportions inégales. L'abondance relative maximale est observée en S7, elle est de l'ordre de 46,01\% (soit 485 individus). On note également que les stations proches des sources de pollution renferment abondamment de Diptères par rapport aux autres stations.

\section{Les Odonates}

Selon les résultats obtenus, Ces insectes sont irréguliers et peu représentés. Ils comptent 137 individus appartenant à 3 familles monospécifiques: Aeshnidae (Aeshna sp.), Gomphidae (Gomphus sp.) et Lestidae (Lestes sp.) La famille la plus abondante est celle des Aeshnidae, elle représente $63,5 \%$ du total des captures (soit 87 individus), les Gomphidae et Lestidae occupent la deuxième et la troisième place des Odonates sur le plan d'abondance numérique. La famille des Lestidae représente $23,35 \%$ (soit 32 individus) et la famille des Gomphidae $13,13 \%$ (soit 18 individus) (Figure 13A). La répartition en individus des Odonates dans les différentes stations étudiées (Figure 13B) met en évidence la faible abondance de ces invertébrés dans la majorité des stations. En revanche, au niveau de S11, on observe un pic maximal de 315 individus (soit $24,38 \%$ de la faune totale inventoriée).
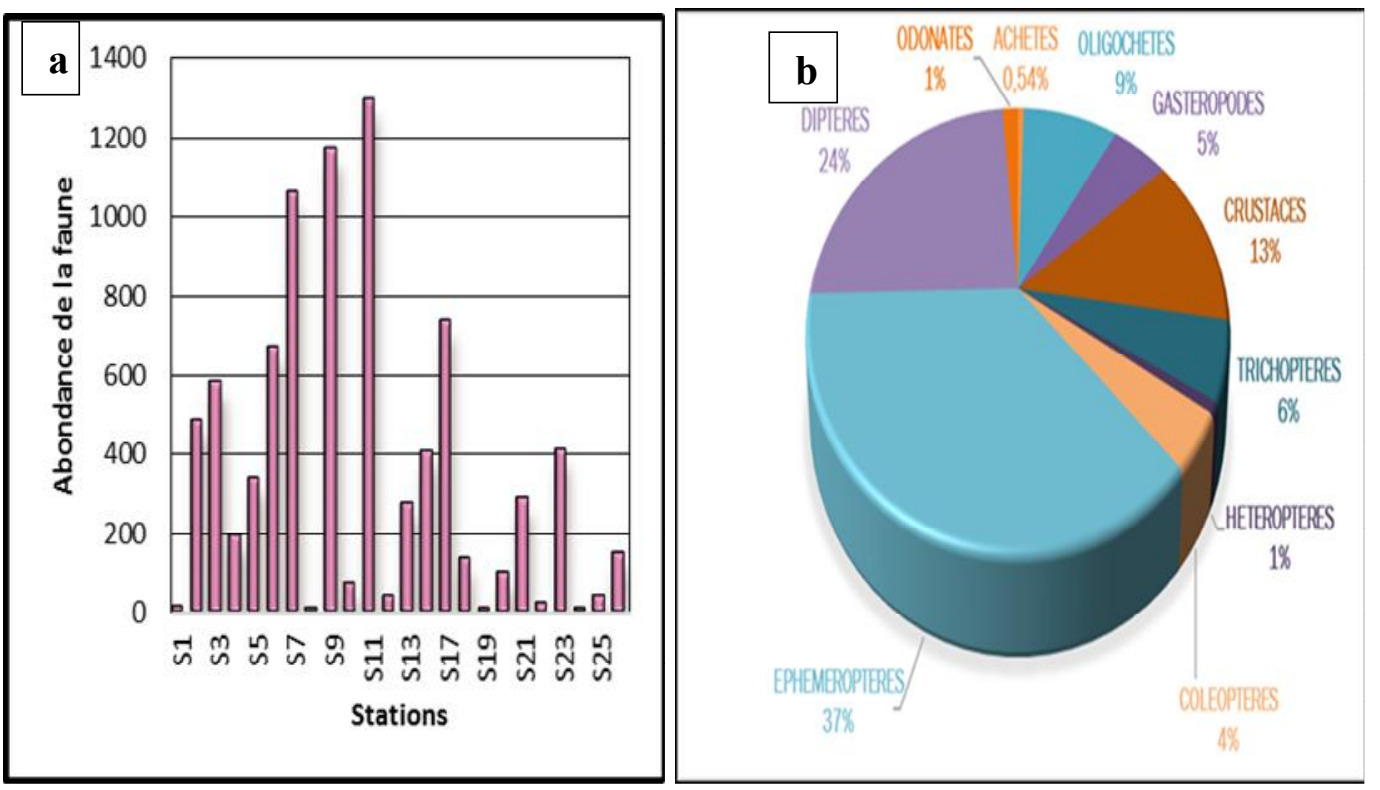

Figure 2 : Distribution de la faune globale (a) et abondance globale (b) dans les eaux des différentes stations étudiées. 
Tableau 2 : Inventaire faunistique globale des peuplements benthiques recensés dans les cours d'eau du réseau hydrographique du bassin du Sebou.

\begin{tabular}{|c|c|c|c|c|c|}
\hline \multicolumn{6}{|c|}{ UNITES SYSTEMATIQUES } \\
\hline Phylum & Classes & Ordre & Famille & Genre & Espèce \\
\hline \multirow[t]{12}{*}{ ANNELIDES } & OLIGOGHETES & HAPLOTAXIDES & Haplotaxidae & Haplotaxis & $\begin{array}{l}\text { Haplotaxis gordioides } \\
\text { (Hartman) }\end{array}$ \\
\hline & & & Lumbricidae & Eiseniella & Eiseniella tetraedra (Savigny) \\
\hline & & & Tubificidae & Tubifex & Tubifex tubifex (Müller) \\
\hline & & & & Aulodrilus & Aulodrilus pluriseta (Piguet) \\
\hline & ACHETES & RHYNCHOBDELLAE & Glossiphoniidae & Glossiphonia & Glossiphonia sp. \\
\hline & & & & Batrocobdella & $\begin{array}{l}\text { Batrocobdella paludosa } \\
\text { (Carena) }\end{array}$ \\
\hline & & & & & $\begin{array}{l}\text { Boreobdella verrucata } \\
\text { ((Müller) }\end{array}$ \\
\hline & & & Erpobdellidae & Erpobdella & Erpobdella octoculata (Linné) \\
\hline & & & & & Erpobdella testacea (Savigny) \\
\hline & & & & Helobdella & $\begin{array}{l}\text { Helobdella stagnalis } \\
\text { (Linnaeus) }\end{array}$ \\
\hline & & & & Haementeria & Haementeria costata (Müller) \\
\hline & & ARHYNCHOBDELLAE & Hirudidae & Hirudo & Hirudo medicinalis (Linné) \\
\hline \multirow[t]{8}{*}{ MOLLUSQUES } & GASTEROPODES & PULMONES & Physidae & Aplexa & Aplexa hypnorum (Linnæus) \\
\hline & & & & Physa & Physa acuta (Draparnaud) \\
\hline & & & Bithyniidae & Bithynia & Bithynia sp. \\
\hline & & & Amnicolidae & Bythinella & Bythinella sp. \\
\hline & & & Planorbidae & Planorbis & Planorbis sp. \\
\hline & & & & Menetus & Menetus dilatatus (Gould) \\
\hline & & & Melanopsidae & Melanopsis & Melanopsis cariosus (Linné) \\
\hline & & PROSOBRANCHES & Viviparidae & Viviparus & Viviparus sp. \\
\hline
\end{tabular}


F. ABBOU et A. FAHDE / Int. J. Biol. Chem. Sci. 11(4): 1785-1806, 2014

\begin{tabular}{|c|c|c|c|c|c|}
\hline & & & Hydrobiidae, & Mercuria & Mercuria similis (Draparnaud) \\
\hline \multirow[t]{24}{*}{ ARTHROPODES } & CRUSTACÉS & AMPHIPODES & Gammaridae & Gammarus & Gammarus sp. \\
\hline & & & & & Gammarus pulex (Gauthieri) \\
\hline & & DECAPODES & Atyidae & Atyaephyra & $\begin{array}{l}\text { Atyaephyra desmarestii } \\
\text { (Milet) }\end{array}$ \\
\hline & INSECTES & TRICHOPTËRES & Hydropsychidae & Hydropsyche & $\begin{array}{l}\text { Hydropsyche maroccana } \\
\text { (Navas) }\end{array}$ \\
\hline & & & Hydroptilidae & Hydroptila & Hydroptila sp. \\
\hline & & & & Orthotrichia & Orthotrichia sp. \\
\hline & & & Philopotamidae & Chiamarra & Chiamarra marginata (Linné) \\
\hline & & & Polycentropodidae & Neureclipsis & $\begin{array}{l}\text { Neureclipsis bimaculata } \\
\text { (Linnaeus) }\end{array}$ \\
\hline & & & Ecnomidae & Ecnomus & Ecnomus sp. \\
\hline & & HÉTÉROPTÈRES & Corixidae & Micronecta & Micronecta scholtzi (Fieber) \\
\hline & & & Notonectidae & Notonecta & Notonecta maculata \\
\hline & & & Gerridae & Gerris & Gerris argentatus (Schummel) \\
\hline & & COLÉOPTÈRES & Haliplidae & Haliplus & Haliplus sp. \\
\hline & & & & Peltodytes & Peltodytes sp. \\
\hline & & & Gyrinidae & Aulonogyrus & Aulonogyrus sp. \\
\hline & & & & Gyrinus & Gyrinus sp. \\
\hline & & & Dytiscidae & Laccophilus & Laccophilus sp. \\
\hline & & & & Agabus & Agabus sp. \\
\hline & & & & Hydroporus & Hydroporus sp. \\
\hline & & & Noteridae & Noterus & Noterus $s p$. \\
\hline & & & Hydrophilidae & Hydrophilus & Hydrophilus sp. \\
\hline & & & & Hydrochara & Hydrochara sp. \\
\hline & & & & Hydrous & Hydrous sp. \\
\hline & & & Elmidae & Limnius & Limnius sp. \\
\hline
\end{tabular}


F. ABBOU et A. FAHDE / Int. J. Biol. Chem. Sci. 11(4): 1785-1806, 2014

\begin{tabular}{|c|c|c|c|}
\hline & Hygrobiidae & Hygrobia & Hygrobia sp. \\
\hline EPHÉMÉROPTÈRES & Baetidae & Baetis & Baetis rhodani (Pictet) \\
\hline & & & Baetis fuscatus (Linnaeus) \\
\hline & & Acentrella & Acentrella sinaica \\
\hline & & Procloeon & Procloeon sp. \\
\hline & & Centroptilum & Centroptilum sp. \\
\hline & Caenidae & Caenis & Caenis luctuosa (Bürmeister) \\
\hline & Leptophlebiidae & Leptophlebia & Leptophlebia sp. \\
\hline & Heptageniidae & Ecdyonurus & $\begin{array}{l}\text { Ecdyonurus rothschildi } \\
\text { (Navas) }\end{array}$ \\
\hline & & Cloeon & Cloeon sp. \\
\hline & & Epeorus & Epeorus sp. \\
\hline & & Rhithrogena & Rhithrogena sp. \\
\hline & Oligoneuriidae & Oligoneuriella & $\begin{array}{l}\text { Oligoneuriella rhenana } \\
\text { (Imhoff) }\end{array}$ \\
\hline DIPTÈRES & Chironomidae & & \\
\hline & Ss-F. Chironominae & & \\
\hline & Tribu : Chironomini & Chironomus & $\begin{array}{l}\text { Chironomus halophilus } \\
\text { (Kieffer) }\end{array}$ \\
\hline & & & Chironomus plumosus (Linné) \\
\hline & & & Chironomus thummi (Kieffer) \\
\hline & & & Chironomus sp. \\
\hline & $\begin{array}{l}\text { Ss-Famille } \\
\text { Orthocladiinae }\end{array}$ & Cricotopus & $\begin{array}{l}\text { Cricotopus annulator } \\
\text { (Goetghebuer) }\end{array}$ \\
\hline & & & Cricotopus bicinctus (Meigen) \\
\hline & & & Cricotopus omatus (Meigen) \\
\hline & & & Cricotopus pallidipes \\
\hline
\end{tabular}


F. ABBOU et A. FAHDE / Int. J. Biol. Chem. Sci. 11(4): 1785-1806, 2014

\begin{tabular}{|c|c|c|c|}
\hline & & & (Edwards) \\
\hline & & & $\begin{array}{l}\text { Cricotopus } \\
\text { (Fabricius) }\end{array}$ \\
\hline & & & Cricotopus sp. \\
\hline & & Psectrocladius & Psectrocladius sp. \\
\hline & Ceratopogonidae & Culicoides & Culicoides sp. \\
\hline & Culicidae & Coquillettidia & Coquillettidia sp. \\
\hline & & Culicinae & Culicinae sp. \\
\hline & Dixidae & Dixa & Dixa sp. \\
\hline & Psychomyiidae & Metalype & Metalype fragilis (Pictet) \\
\hline & Limoniidae & Dicranota & Dicranota sp. \\
\hline & Ptychopteridae & & \\
\hline & Psychodidae & & \\
\hline & Simuliidae & Whilhelmia & $\begin{array}{l}\text { Whilhelmia pseudoquinum } \\
\text { (Seguy) }\end{array}$ \\
\hline & & Metacnephia & $\begin{array}{l}\text { Metacnephia blanci (Grenier \& } \\
\text { Theodorides) }\end{array}$ \\
\hline & Stratiomyidae & & \\
\hline & Syrphidae & & \\
\hline & Anthomyidae & & \\
\hline & Tabanidae & & \\
\hline & Tipulidae & & \\
\hline ODONATES & Aeshnidae & Aeshna & Aeshna sp. \\
\hline & Gomphidae & Gomphus & Gomphus sp. \\
\hline & Lestidae & Lestes & Lestes sp. \\
\hline
\end{tabular}




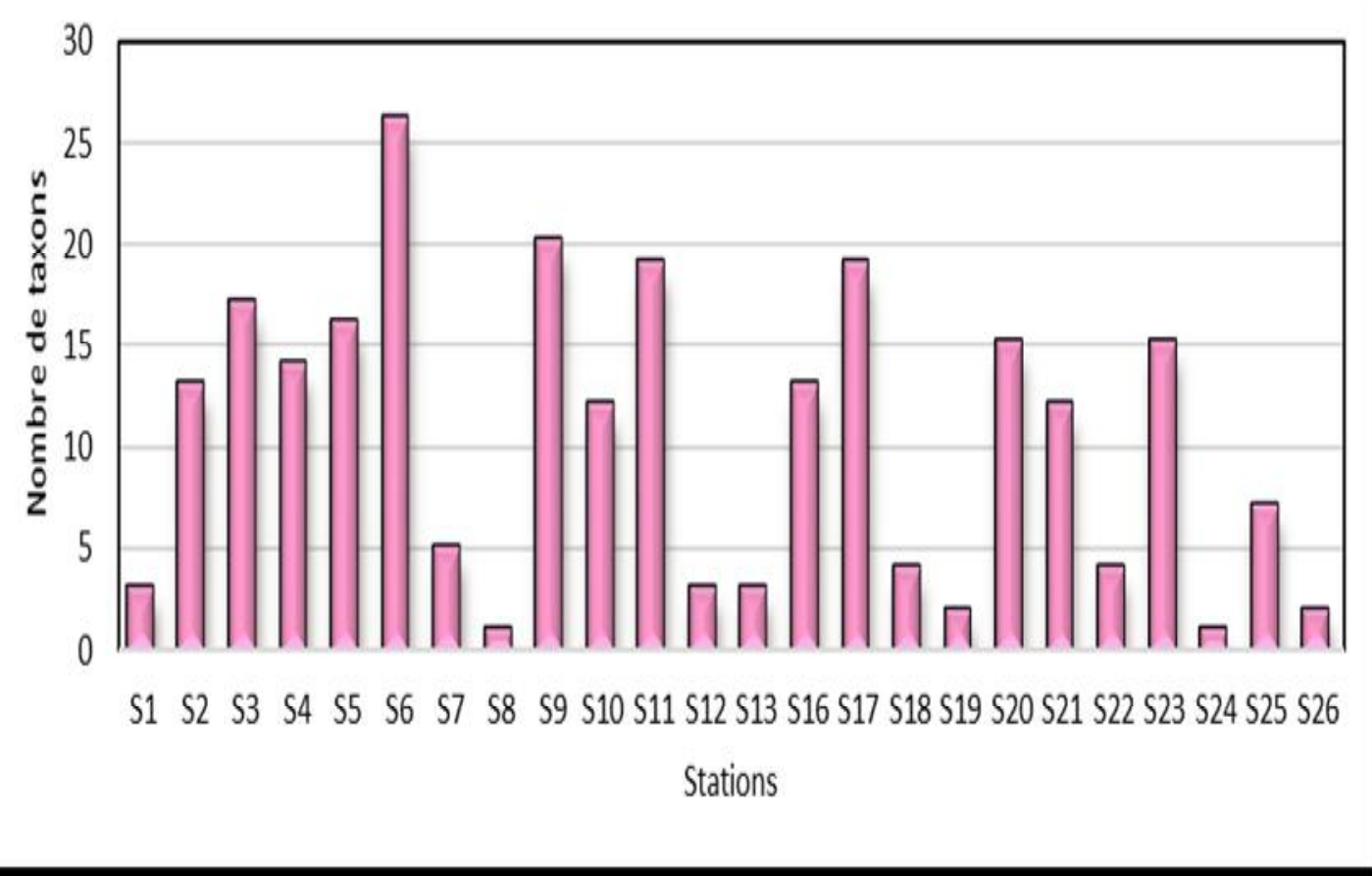

Figure 3 : Evolution spatiale des effectifs totaux des taxons dans les différentes stations étudiées.
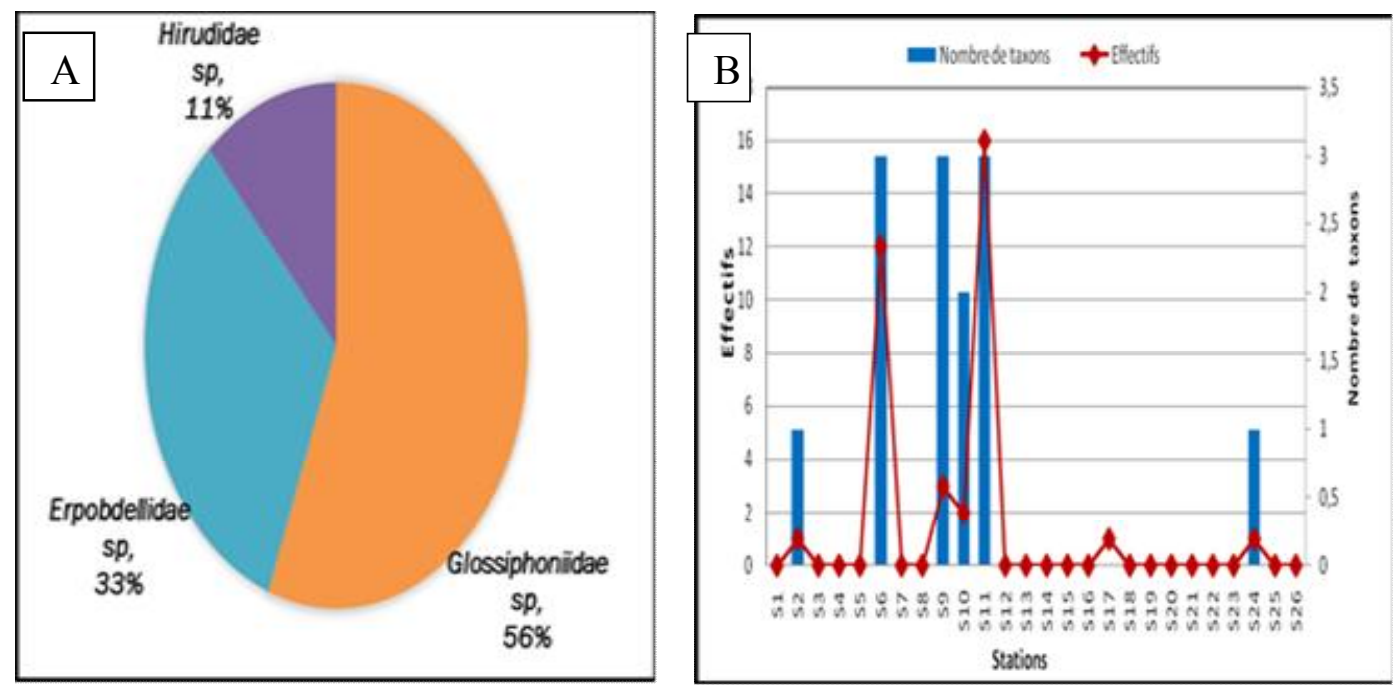

Figure 4 : Abondance (A) et répartition spatiale (B) des Achètes dans les stations d'étude. 

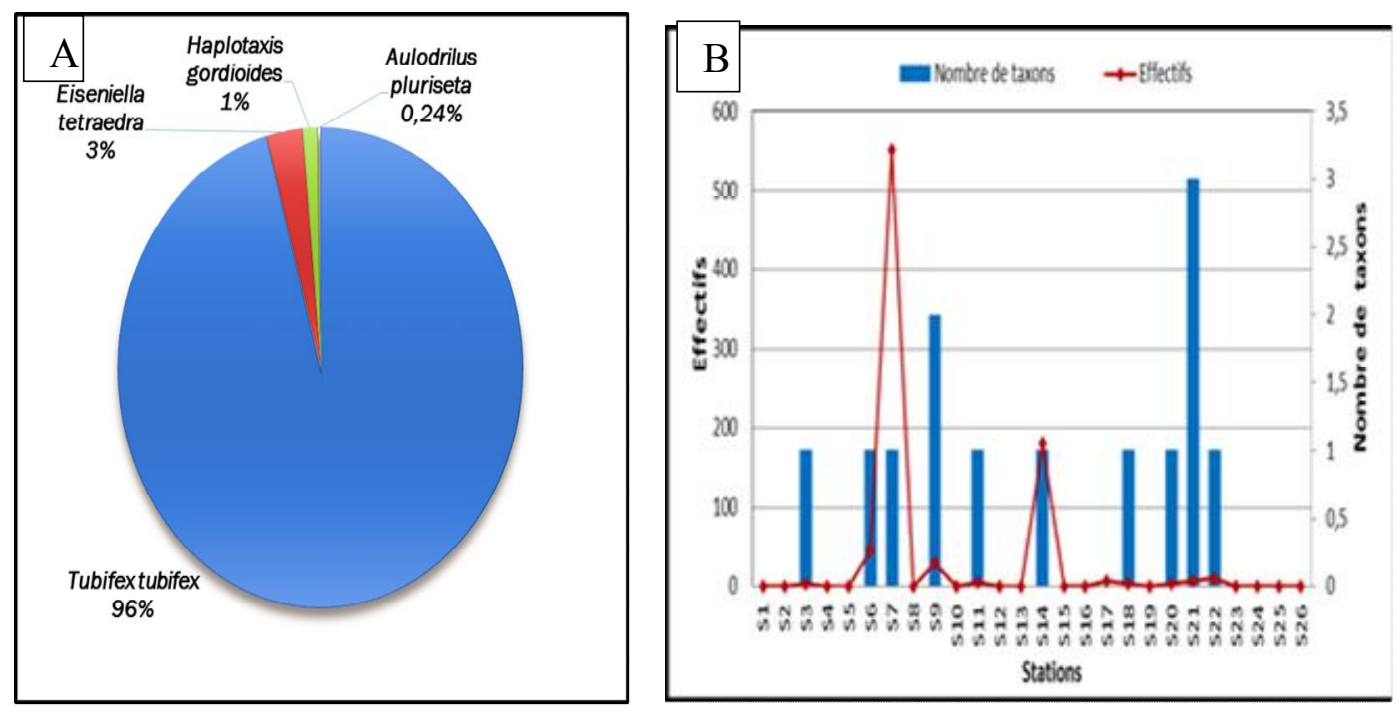

Figure 5 : Abondance (A) et répartition spatiale (B) des Oligochètes dans les stations d'étude.
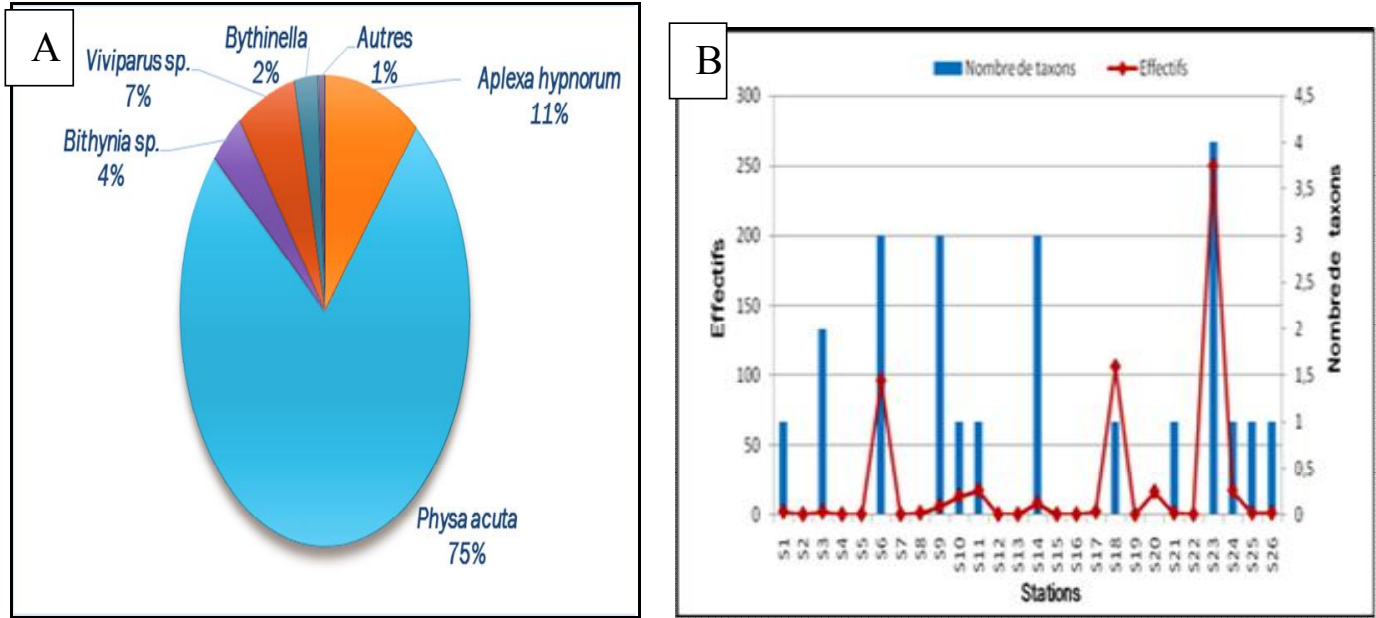

Figure 6 : Abondance (A) et répartition spatiale (B) des Gastéropodes dans les stations d'étude. 

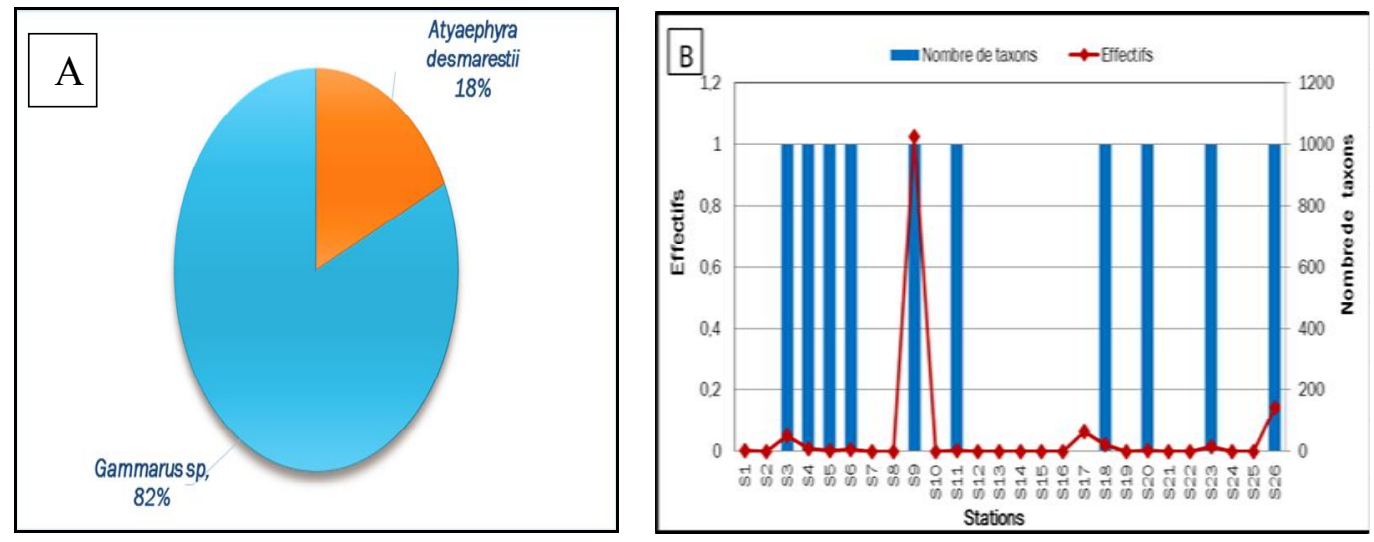

Figure 7 : Abondance (A) et répartition spatiale (B) des Crustacés dans les stations d'étude.
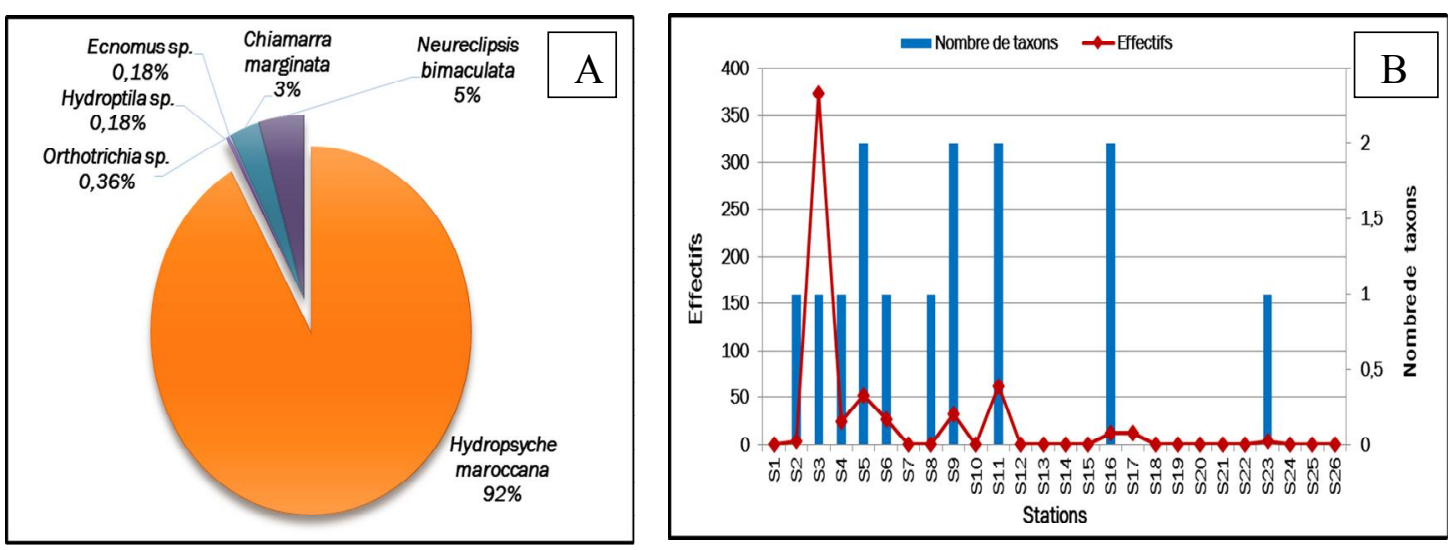

Figure 8 : Abondance (A) et répartition spatiale (B) des Trichoptères dans les stations d'étude.
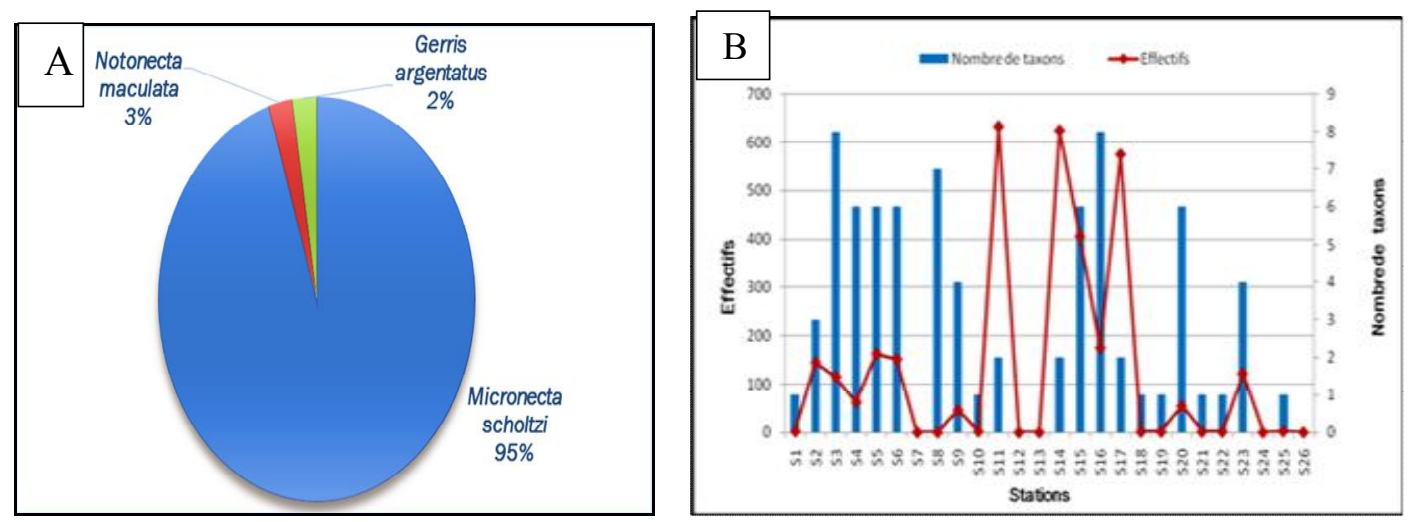

Figure 9 : Abondance (A) et répartition spatiale (B) des Hétéroptères dans les stations d'étude. 

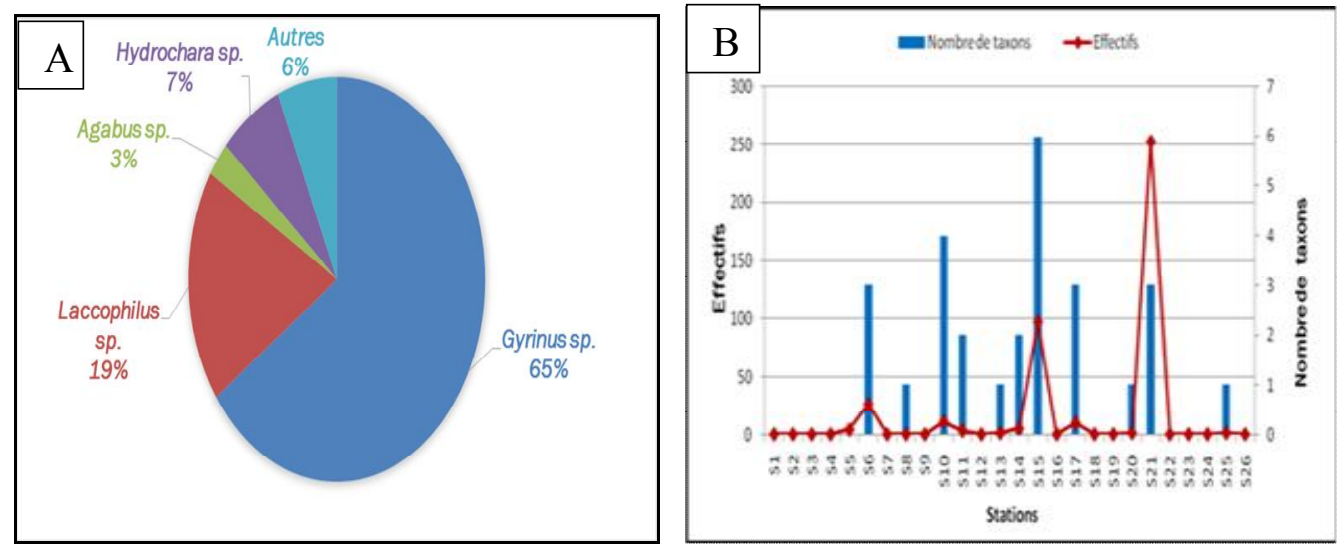

Figure 10 : Abondance (A) et répartition spatiale (B) des Coléoptères dans les stations d'étude.
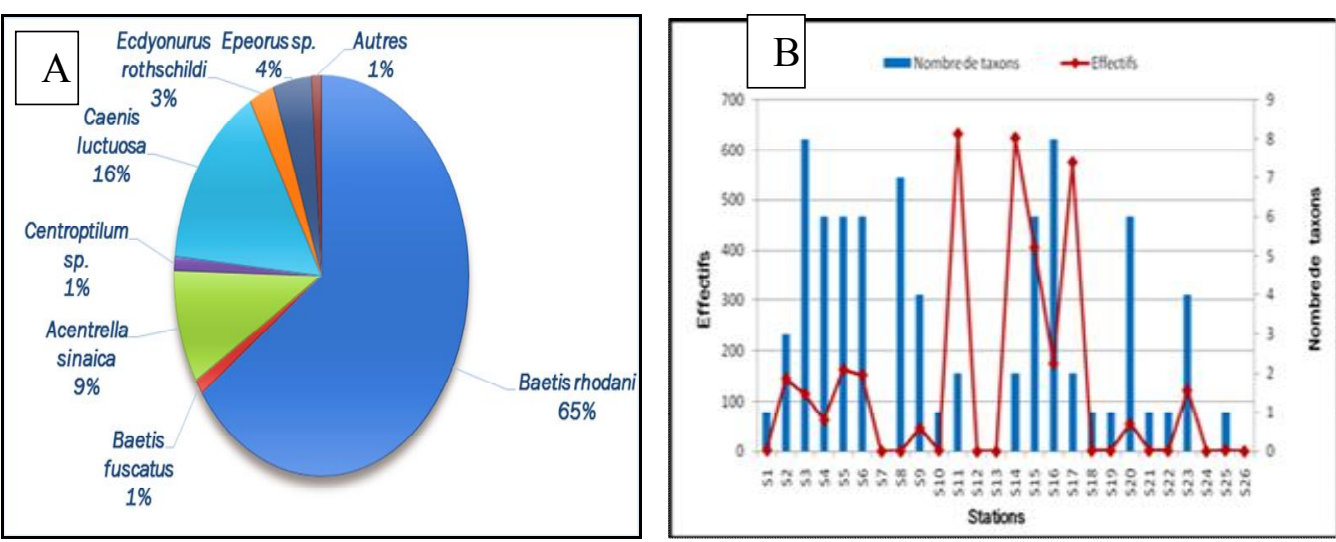

Figure 11 : Abondance (A) et répartition spatiale (B) des Ephéméroptères dans les stations d'étude.
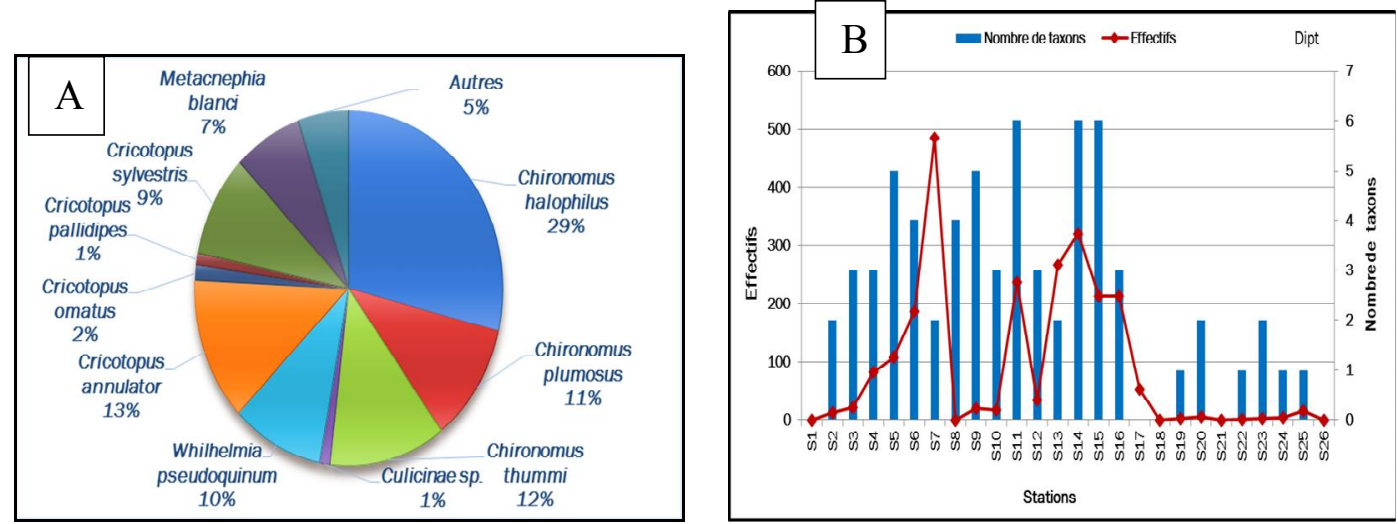

Figure 12 : Abondance (A) et répartition spatiale (B) des Diptères dans les stations d'étude. 

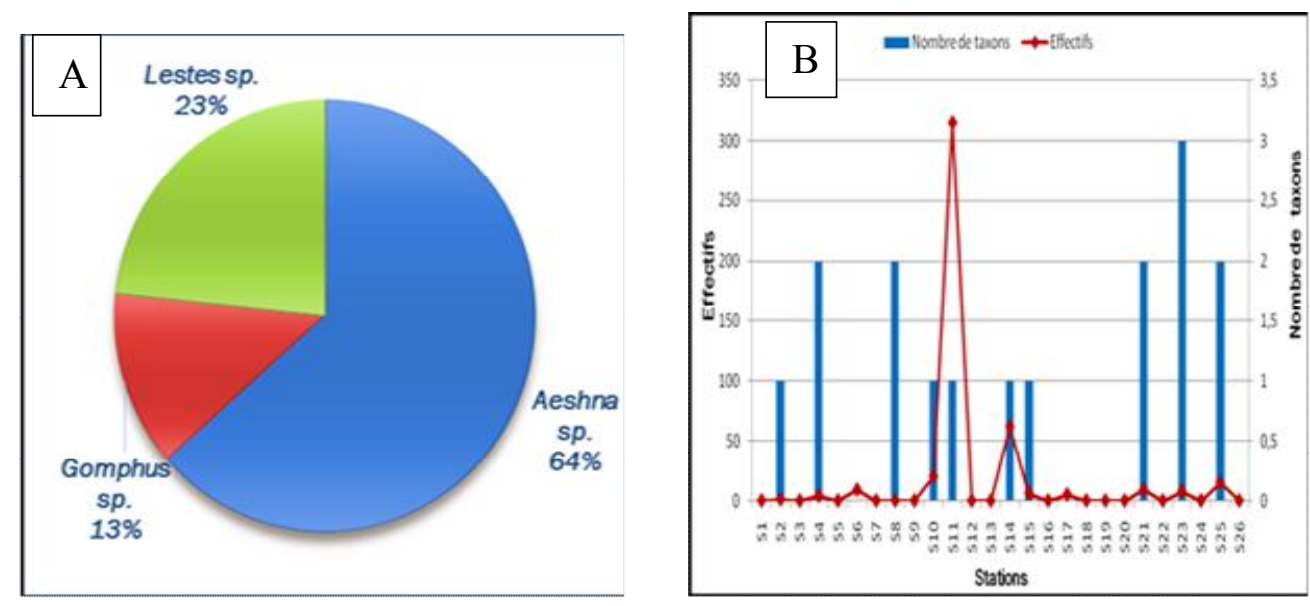

Figure 13 : Abondance (A) et répartition spatiale (B) des Odonates dans les stations d'étude.

\section{DISCUSSION}

L'appauvrissement en taxons du bassin du Sebou serait dû aux particularités hydrochimiques des milieux étudiés. Ce même résultat a été obtenu par Karrouch (2010). Selon (Oualad Mansour et al., 2009), une forte minéralisation entraine une réduction de la diversité spécifique dans un peuplement benthique. En effet, les fortes valeurs de conductivité $(3020$ à $8000 \mu \mathrm{s} / \mathrm{cm})$ ont entrainé la disparition des Plécoptères ainsi que certaines espèces d'Ephéméroptères et de Trichoptères et donc un appauvrissement de la diversité des communautés benthiques.

Dans la zone étudiée, l'absence naturelle de couvert végétal et sa dégradation augmentent les écarts thermiques saisonniers. Ces contraintes thermiques expliqueraient aussi l'appauvrissement de la faune des cours d'eau étudiés (Oubraim, 2002; Agblonon Houelome, 2016). Aussi, Ben moussa (2012) signale qu'un appauvrissement taxonomique graduel, affectant tous les groupes zoologiques s'observe suivant l'axe Nord-Sud depuis l'Europe jusqu'en Afrique du Nord, L'effet conjugué des fortes températures et de la salinité accentue le phénomène jusqu'à des peuplements appauvris à l'extrême (Tachet et al., 2000).

La majorité des taxons inventoriés ont, de point de vue biogéographique, une origine paléarctique; quelques rares taxons ont, par contre, une origine éthiopienne.

A ce propos, les résultats de la présente étude rejoignent ceux obtenus par Oubraim (2002). En effet, de par leur situation géographique, ces différents taxons se trouvent à la limite Sud de leur aire de distribution au sein de la zone paléarctique et subissent des changements saisonniers accentués ce qui entraine une réduction de la diversité taxonomique. Aussi, les eaux des stations situées à proximité des agglomérations sont fortement minéralisées et chargées en matière organique. Le stress dans ce milieu est donc sévère et serait à l'origine de la réduction de la composition de la communauté benthique. Ainsi, un cours d'eau perturbé peut créer des conditions défavorables pour certains organismes (polluo-sensibles) laissant la place ainsi à d'autres organismes plus tolérants (polluorésistants) (Fagrouch et al., 2011). Dans les stations étudiées, les taxons dominants sont les Diptères (essentiellement les Chironomidae) et les Annélides (essentiellement les Oligochètes). Des constats similaires ont été relevés dans les travaux de Oubraim (2002) sur l'oued Mellah et Ben moussa (2014) sur l'oued Khoumane.

Concernant les autres stations, elles montrent globalement une variété 
taxonomique plus importante. Cette évolution des effectifs totaux des taxons pourrait s'expliquer par l'éloignement de la source de pollution et donc une possibilité de récupération de la qualité des eaux au niveau de certaines zones.

De façon générale, on constate, au niveau des stations étudiées, une dominance des taxons polluo-résistants, principalement les Diptères et les Annélides. Ce résultat est lié au degré de pollution organique élevé en aval des agglomérations et qui est favorable à la prolifération de ces invertébrés polluorésistants (De Moor et al., 2002). Les Ephéméroptères occupent presque la totalité des milieux étudiés, avec des abondances relativement importantes. Ce résultat est lié à la nature de ces habitats caractérisés par des températures relativement élevées et un substrat hétérogène riche en matière organique, offrant des conditions fortement favorables au développement d'une faune plus abondante, surtout les Baetidae qui sont des Ephéméroptères qui supportent la pollution (Haouchine, 2011).

Les Coléoptères constituent un groupe plus au moins diversifié par rapport aux autres groupes. En effet, ils sont les seuls insectes holométaboles à se présenter à la fois sous la forme imaginale et sous la forme larvaire dans les milieux aquatiques. Ils colonisent divers habitats : sources, ruisseaux de sources, rivières à eau modérément courante et rivières à eau quasi-stagnante et riche en végétation (Tachet et al., 2010). L'abondance relativement importante des Trichoptères, représentés essentiellement par Hydropsyche maroccana, est dû au fait que ces invertébrés ont une résistance relative à la pollution.

Concernant les Odonates et les Gastéropodes, ils sont irréguliers et peu représentés. En effet, la répartition et la prolifération des mollusques dans les eaux continentales sont influencées par plusieurs facteurs : la végétation, la teneur en calcium, la nature du substrat et la vitesse du courant d'eau (Belqat et al., 2004 ; Karrouch, 2010 ; Ouedraogo et al., 2015). Par contre, on note une absence totale des Plécoptères. Ce même résultat a été obtenu dans des travaux antérieurs au niveau de l'oued Boufekrane (Aboulkacem, 2007), au niveau de l'oued Mellah (Oubraim, 2002) et au niveau de l'Oued Ouislane (Aboulkacem, 2007). Ceci pourrait s'expliquer par le fait que les Plécoptères sont des invertébrés polluosensibles aux milieux affectés par quelconque perturbation (Haouchine, 2011).

Comme déjà noté par Tachet (2006) et Foto Menbohan (2012), le phénomène de l'anthropisation de l'Oued, pourrait être à l'origine d'une disparition des taxons polluosensibles (tels que les Plécoptères) et de la prolifération des groupes polluo-tolérants tels que les Chironomidae.

$\mathrm{La}$ richesse taxonomique varie considérablement d'une station à l'autre. En effet, elle dépend des conditions écologiques ; un habitat hétérogène, offrant une variété de niches écologiques, favorise la prolifération de différents taxons. En revanche, plus le degré de pollution est élevée plus le cours d'eau est pauvre en faune, cas des cours d'eau du bassin du Sebou.

Les faibles richesses spécifiques des communautés benthiques du bassin étudié sont en accord avec ce que certains chercheurs, dont (Moisan et al., 2008) ainsi que (Diomande et al., 2000), appellent « paradoxe des eaux saumâtres ».

\section{Conclusion}

La faune totale inventoriée dans la présente étude compte 9990 individus correspondant à 52 familles et à 80 taxons appartenant à 3 groupes faunistiques (Annélides, Mollusques, Arthropodes). Dans un but comparatif des structures des communautés benthiques étudiées, on a utilisé les abondances relatives des différents taxons. Selon les résultats obtenus, les Ephéméroptères, les Diptères, les Crustacés et les Oligochètes sont dominants. Les autres groupes faunistiques ne constituent qu'une faible fraction de la faune récoltée. Les taxons Baetis rhodani (2299 individus), Chironomus 
sp. (1205 individus), Gammarus sp. (1025 individus), Tubifex tubifex (802 individus) sont les plus numériquement inventoriés. Cependant, on note une absence totale du groupe polluosensible : les Plécoptères. Ceci révèle la situation critique des eaux du bassin, due essentiellement au phénomène de l'anthropisation de l'Oued. La richesse taxonomique varie considérablement d'une station à l'autre. En effet, les stations situées en aval des zones les plus urbanisées et industrialisées soumises aux rejets domestiques et industrielles se caractérisent par le développement de certains groupes zoologiques polluo-résistants, principalement les Oligochètes et les Diptères, au détriment des autres groupes. Par contre, les autres stations, éloignées des sources de pollution, se caractérisent par une réapparition de certains taxons qui tolèrent une certaine charge organique, tels que les Ephéméroptères (Baetis sp.), les Crustacés (Gammarus sp. et Athyaephyra desmarestii), les Trichoptères (Hydropsyche maroccana).

L'exposition des eaux du bassin hydrographique du Sebou aux différentes sources de pollution contribue ainsi, à l'installation de conditions défavorables à la présence d'un peuplement très diversifié.

\section{CONFLIT D'INTERETS}

Les auteurs déclarent qu'il n'y a aucun conflit d'intérêt.

\section{CONTRIBUTIONS DES AUTEURS}

FA a procédé à la collecte des données, à l'analyse des données et à la rédaction du manuscrit ; AF a suivi l'exécution du protocole, a procédé à l'analyse des données, à la lecture et à la correction du manuscrit.

\section{REMERCIEMENTS}

Les auteurs remercient l'équipe du Laboratoire de Santé et d'Environnement de la Faculté des Sciences Ain Chock de l'Université Hassan II à Casablanca (Maroc) pour leur appui technique et leurs efforts dans la collecte des données.

\section{REFERENCES}

Aboulkacem A. 2007. Etude hydrobiologique comparative des oueds Boufekrane et Ouislane à la traversée de la ville de Meknès. Impact sur la Sante et l'Environnement. Thèse d'Etat, Facultés des Sciences, Université Moulay Ismail, Meknès (Maroc), p. 159.

Agblonon Houelome TM, Adandedjan D, Chikou A, Imorou Toko I, Bonou C, Youssao I, Laleye P. 2016. Evaluation de la qualité des eaux des ruisseaux du cours moyen de la rivière Alibori par l'étude des macroinvertébrés benthiques dans le bassin cotonnier du Bénin (Afrique de l'Ouest). Int. J. Biol. Chem. Sci., 10(6): 2461-2476. DOI: http://dx.doi.org/10.4314/ijbcs.v10i6.5

Barton DR, Metcalf Smith JL. 1992. A comparison of sampling techniques and summary indices for assessment of water quality the Yamaska river, Quebec, based on benthic macroinvertebrates. Environ. Monit. And Assess., 21: 225244. DOI: https://doi.org/10.1007/ BF00399689

Belqat B, Dakki, M. 2004. Clés analytiques des Simulies (Diptera) du Maroc. Zoologica Baetica, 15: 77-137.

Ben moussa A, Chahlaoui A, Rour E. 2012. Evaluation de la pollution physicochimique des eaux de l'Oued Khoumane (Moulay Idriss Zerhoun, Maroc). Int. J. Biol. Chem. Sci., 6: 70-96. DOI : http://dx.doi.org/10.4314/ijbcs.v6i6.44

Ben Moussa A, Chahlaoui A, Rour E, Chahboune M. 2014. Diversité taxonomique et structure de la macrofaune benthique des eaux superficielles de l'oued khoumane. Moulay idriss Zerhoun, Maroc. J. Mater. Environ. Sci., 5(1): 189 p. DOI : http://eprints.umi.ac.ma/id/eprint/989

Beyene A, Addis T, Kifle D, Legesse W, Kloos H, Triest L. 2009. Comparative study of diatoms and macroinvertebrates as indicators of severe water pollution: Case study of the 
Kabena and Akaki rivers in Addis Abeba, Ethiopia. Ecological Indicator, 9: $381-392 . \quad$ DOI : 10.1016/j.ecolind.2008.05.001

Clergue-Gazeau M. 1991. Clés de détermination des Simulies (Diptera, Simuliidae) des Pyrénées. Annls Limnol. 27(3): $267 . \quad$ DOI: https://doi.org/10.1051/limn/1991020

Colas F, Vigneron A, Felten V, Devin S. 2014. The contribution of a niche-based approach to ecological risk assessment: using macro-invertebrates species under multiple stressors. Environmental Pollution, 185: 24 - 34. DOI : 10.1016/j.envpol.2013.09.033

De Moor IJ, Day JA, De Moor FC. 2002. Guides to the Freshwater Invertebrates of Southern Africa Diptera. Prepared for the Water Research Commission (Vol 9), WRC Report No. TT 214/03: Pretoria; 210.

Diomande D, Gourene G, San Kare Y, Zabi SG. 2000. Synopsis de la classification des larves et des nymphes de diptères chironomidae des écosystèmes dulçaquicoles de l'Afrique de l'Ouest: clés de détermination des sous-familles, des tribus et des genres. Archives Scientifiques, 27: 1. DOI : http://aquaticcommons.org/id/eprint/7488

Dommanget JL. 1994. Atlas préliminaire des Odonates de France. Etat d'avancement au 31/12/1993. Coll. Patrimoines Nationals, Vol. 1,6.- Paris SEF/MNHN, SFO et Min. Env. 80p.

Fagrouch A, Berrahou A, El Halouani H, 2011. Impact d'un effluent urbain de la ville de Taourirt sur la structure des communautés de macroinvertébrés de l'oued Za (Maroc oriental). Revue des Sciences de l'Eau, 24(2) : 87-101. DOI : 10.7202/1006104ar

Foto Menbohan S, Zebaze Togouet SH, Njine T, Nyamsi Tchatcho NL. 2010. Macroinvertébrés benthiques du cours d'eau Nga: Essai de caractérisation d'un référentiel par des analyses biologiques.
European Journal of Scientific Research, 43(1): 96 - 106.

Foto Menbohan S, Zebaze Togouet SH, Nyamsi Tchatcho Nl, Njine T. 2011. Evolution spatiale de la diversité des peuplements de macroinvertébrés benthiques dans un cours d'eau anthropisé en milieu tropical (Cameroun). Eur. J. Sci. Res., 55(2): 291-300.

Foto Menbohan S, Koji E, Ajeagah Gideon A, Bilong Nilong CF, Njiné T, 2012. Impact of dam construction on the diversity of benthic macroinvertebrates community in a periurban stream in Cameroon. International Journal of Biosciences, 11: 137 - 145.

Foto Menbohan S, Tchakonte S, Ajeagah G, Zebaze Togouet SH, Bilong Bilong Cf, Njine T, 2013. Water quality assessment using benthic macroinvertebrates in a periurban stream (Cameroon). International Journal of Biotechnology 2(5): 91-104.

Genin B, Chauvin C, Menard F. 1997. Cours d'Eau et Indices Biologiques: Pollution et Méthodes IBGN. ENESA-CNERTA : Dijon ; 202.

Haouchine S. 2011. Recherche sur la faunistique et l'écologie des macroinvertébrés des cours d'eau de Kabylie. Mémoire de Master, Faculté des Sciences Biologiques et Sciences Agronomiques, Université Mouloud Mammeri de Tizi ouzou, Algérie, 157 pp.

Hynes HBN. 1960. The Biology of Polluted Waters. Liverpool University Press: Liverpool; 202.

Kaboré I, Ouédraogo I, Tampo L, Ouéda A, Moog O, Guenda W, Melcher AH. 2016. Composition and dynamic of benthic macroinvertebrates community in semiarid area rivers of Burkina Faso (West Africa). Int. J. Biol. Chem. Sci., 10(4): 1542-1561.

DOI: http://dx.doi.org/10.4314/ ijbcs.v10i4.8 
Karrouch L. 2010. Bio-évaluation de la qualité des eaux courantes de la région Meknès (Centre- Sud, Maroc) - Impact sur l'environnement et la sante. Thèse doctorat es sciences en biologie. Facultés des Sciences, Université Moulay Ismaïl, Meknès, Maroc, 216 pp.

Lascombe C. 1992. Les méthodes d'évaluation de la qualité des milieux aquatiques superficiels, Tribune de l'Eau. 555 : 18-29.

Moisan J, Pelletier L. 2008. Guide de surveillance biologique basée sur les macroinvertébrés benthiques d'eau douce du Québec - Cours d'eau peu profonds à substrat grossier. Direction du suivi de l'état de l'environnement, ministère du Développement durable, de l'Environnement et des Parcs, 86 p.

Ouedraogo I, Oueda A, Sirima D, Ouedraogo I, Guenda W, Kabré GB. 2015. Assessment of benthic molluscs diversity and distribution in urban reservoirs. Int. J. Biol. Chem. Sci., 9: 2066-2077. DOI: http://dx.doi.org/10.4314/ijbcs.v9i4.29

Oubraim S. 2002. Qualité physico-chimique et biologique des cours d'eau du réseau hydrographique de la meseta occidentale marocaine : cas de l'oued Mellah. Thèse de Doctorat d'Etat Es Science. Fac. Sci. Ben M'Sik Casablanca, 72-73.

Oualad Mansour N, Kamal T, Stitou J. 2009. Evaluation de la qualité des eaux dans les systèmes fluviaux du Rif (cas de la rivière Martil) et étude de la biodiversité des communautés de macroinvertébrés. Segundo congreso internacional sobre geologia y mineria en la ordenación del territorio y en el desarrollo. Utrillas 95114.

Poisson R. 1957. Hétéroptères aquatiques. Faune de la France, 61: 261.

Tachet H, Richoux P, Bournaud M, UsseglioPolatera P. 2000. Invertébrés d'Eau Douce. Systématique, Biologie, Ecologie. CNRS Editions : Paris ; 588.

Tachet H, Richoux P, Bournaud M, UsseglioPolatera P. 2006. Invertébrés d'Eau Douce: Systématique, Biologie, Ecologie (2 ${ }^{\text {ème }}$ Edns. CNRS) : Paris ; 588.

Tachet H, Richoux P, Bournaud M, UsseglioPolatera P. 2010. Invertébrés d'Eau Douce. Systématique, Biologie, Ecologie. CNRS Editions, Paris ; 588. 